\title{
Mortality and tardive dyskinesia: long-term study using the US National Death Index
}

Charles E. Dean and Paul D. Thuras

\section{Background}

Whether the development of tardive dyskinesia leads to an increase in mortality is still unclear.

\section{Aims \\ To explore the relationship between tardive dyskinesia and mortality over a 10-year period, using the National Death Index}

\section{Method}

Death certificates were obtained from the National Death Index on 1621 people repeatedly assessed for tardive dyskinesia by trained raters. Variables with the potential for influencing survival time were also investigated.

\section{Results}

Tardive dyskinesia was significantly associated with an increase in mortality $(P<0.001)$, but this association became non-significant when drug course and age were entered in the regression analysis. Those who had taken only conventional antipsychotics were twice as likely to die compared with those taking atypical agents $(P<0.02)$. For those aged 53-65 years, conventional agents were associated with a sevenfold increase in mortality.

\section{Conclusions}

Older individuals with tardive dyskinesia treated with conventional antipsychotics appear to have a shortened survival time.

\section{Declaration of interest}

None.
Whether the development of tardive dyskinesia is associated with a shortened survival time has been open to question. A recent meta-analysis ${ }^{1}$ of seven studies found that tardive dyskinesia is a weak risk factor for increased mortality, with an odds ratio (OR) in a fixed-effects model of 1.4 (95\% CI 1.2-1.7, $P<0.005)$. However, the authors described recurrent methodological problems, including small sample sizes, a follow-up time of less than 5 years in three studies and varying definitions of tardive dyskinesia. We further note that six studies lacked data on interrater reliability. Despite the advent of atypical antipsychotics, the continuing use of conventional antipsychotics in poorer countries $^{2}$ and in some segments of more economically advantaged areas $^{3}$ makes this question still important, particularly in view of the metabolic risks associated with atypical antipsychotics. ${ }^{4-6}$

\section{Method}

The Minneapolis Veterans Administration Medical Center requires that each patient exposed to antipsychotics be evaluated at least yearly for tardive dyskinesia by raters repeatedly trained to reliability in using the Dyskinesia Identification SystemColdwater version (DIS-CO), ${ }^{7}$ a scale which is equivalent in sensitivity $^{8}$ to the Abnormal Involuntary Movement Scale (AIMS). ${ }^{9}$ Dyskinesia Identification System scores were reviewed by the attending physician who had to reach a conclusion regarding the presence or absence of tardive dyskinesia based on the Schooler-Kane criteria. ${ }^{10}$ Depending on staffing, only two to three trained raters were used during this study, with $\kappa$ coefficients ranging from 0.80 to 0.85 . Demographic data, smoking history, Axis I and Axis III diagnoses, ${ }^{11}$ names and doses of medications extant at the time of each rating, and previous DIS-CO scores were entered in a computerised database. A waiver of authorisation was obtained from the Minneapolis Veterans Administration Medical Center Human Studies Subcommittee to submit detailed identification data on 1626 patients to the National Death Index. ${ }^{12}$ Observation times ranged from 3 to 5074 days, with a mean observation time of 1446 days (s.d.=1179.3). The National Death Index, after ensuring that this study met their standards with regard to confidentiality and appropriate use of death certificate data, supplied us with certificates noting the causes of death in 1200 people. Pilot data had indicated that 24 of 386 people had died over a 10-year period. A power analysis indicated that utilisation of the complete database would give an $87 \%$ likelihood of detecting differences in mortality at a moderate effect size.

\section{Data analysis}

With survival time as the censored, failure-time, dependent variable, we used the Cox proportional hazards regression, a multivariate extension of the Kaplan-Meier method, to estimate the relative risk of mortality associated with several predictor variables, including time since last examination, age, the diagnosis of tardive dyskinesia (ever or current), course of antipsychotic use (conventional antipsychotics only, atypical antipsychotics only or a mix), and the interaction of tardive dyskinesia diagnosis with drug course. For these analyses the observation period was from the date of the last examination to death or the censor period of 31 December 2001.

\section{Results}

The study population (Table 1) was predominantly male (95\%), White (91\%) and African-American (6.6\%). Overall, 60\% had received only conventional antipsychotics and 23\% received both atypical antipsychotics and conventional antipsychotics or switched use. A third developed tardive dyskinesia at some point during follow-up. Of the 1208 individuals with definitive mortality data from the National Death Index, 205 (17.1\%) had died during the observation period. The mean time to death was 1030.1 days $($ s.d.=976.9) from the initial observation $v .1551$ days (s.d.=1202.9) for those alive during the same period. The mortality rate was 5.07 per 100 patient years. 


\begin{tabular}{|ll|}
\hline Table 1 Characteristics of the sample & \\
\hline & $\begin{array}{l}\text { Sample } \\
(n=1208)\end{array}$ \\
\hline Characteristics & 95.5 \\
\hline Men, \% & $49.2(14.0)$ \\
\hline Age at first observation, years: mean (s.d.) & $53.7(13.6)$ \\
\hline Age at last observation, years: mean (s.d.) & \\
\hline Ethnicity, \% & 91.0 \\
White & 6.6 \\
African-American & 1.0 \\
Latino & 0.3 \\
Asian & 0.1 \\
\hline Native American & 60.8 \\
\hline Drug course, \% & 16.1 \\
Conventional antipsychotic & 23.0 \\
\hline Atypical antipsychotic & 33.4 \\
Dual or switched use & 3.84 (3.63) \\
\hline Tardive dyskinesia & 5.29 (4.66) \\
Any, \% & \\
DIS-CO score, mean (s.d.) & \\
Maximum DIS-CO score, mean (s.d.) & \\
DIS-Co, Dyskinesia Identification System-Coldwater version. & \\
\hline
\end{tabular}

\section{Individual factors associated with mortality}

Risk ratios and associated statistics for individual predictors are shown in Table 2. On the initial analysis, without covariates, the presence of tardive dyskinesia, either current or remitted, was significantly associated with an increase in mortality, with a risk ratio (RR) of 1.57 (95\% CI 1.23-1.99, $P<0.001)$. A parallel analysis was conducted in which the maximum DIS-CO score was used, rather than the tardive dyskinesia diagnosis. We found that for every point increase in the DIS-CO score, there was a $5 \%$ increase in the likelihood of death $(\mathrm{RR}=1.05,95 \%$ CI 1.03-1.07, $P<0.001)$.

\section{Age}

We grouped the cohort into four categories: age $<44$ (the reference category), 44-52, 53-65, and 66 years and older. We found that age at the time of the last rating was significantly associated with mortality. The risk ratios were 1.70 (95\% CI 0.99-2.93), 4.55 (95\% CI 2.84-7.30) and 9.06 (95\% CI 5.82-14.09) respectively.

\section{Antipsychotic medication}

For drug course, those who had taken conventional antipsychotics or conventional antipsychotics plus atypical antipsychotics were compared with those who had taken only atypical antipsychotics (the reference category). Those who were exposed only to conventional antipsychotics were over twice as likely to die during the observation period as those who had taken only atypical antipsychotics $(\mathrm{RR}=2.00,95 \% \mathrm{CI}=1.13-3.53, P<0.02)$.

Given that atypical antipsychotics had been less widely used during the early period of this study, we examined the possibility of an interaction between age and drug course, but this was not significant. In a further analysis of drug course and mortality across age strata, we noted that in the age group $>44$ years, the risk ratio for those taking conventional antipsychotics $v$. atypical antipsychotics was 2.2 (95\% CI $0.3-17.4)$, for the group aged 44-52 years the $\mathrm{RR}=2.1$ (95\% CI 0.5-9.0), for those aged 53-65 the $\mathrm{RR}=2.9$ (95\% CI $0.71-12.1$ ), and in those 66 years and older the $\mathrm{RR}=1.8(95 \%$ CI $0.74-4.5)$.

\section{Cox proportional hazards regression analysis}

We entered the presence of tardive dyskinesia, drug course and age into a regression model (final regression model shown in Table 3). Age and drug course continued to be significantly associated with a shortened survival time, but tardive dyskinesia was no longer significant. A parallel analysis using the maximum DIS-CO score yielded similar results. An interaction term with tardive dyskinesia status and drug course was not significant.

\section{Tardive dyskinesia and causes of death}

Using a broad classification of causes of death, we found that $23.9 \%$ had died of cardiac disease/myocardial infarction, $20.5 \%$ from cancer and $8.3 \%$ from stroke/cardiovascular disease. Death by suicide was found in $6.8 \%$ and $4.9 \%$ had died in accidents

\begin{tabular}{|c|c|c|c|c|c|c|}
\hline Variable & Coefficient & s.e. & Wald & $P$ & $\mathrm{RR}$ & $95 \% \mathrm{Cl}$ \\
\hline $\begin{array}{l}\text { Diagnosis of tardive dyskinesia } \\
\text { (present or remitted) }\end{array}$ & 0.45 & 0.12 & 13.26 & $<0.001$ & 1.57 & $1.23-1.99$ \\
\hline DIS-CO score (maximum) & 0.05 & 0.01 & 20.72 & $<0.001$ & 1.05 & $1.03-1.07$ \\
\hline Use of conventional antipsychotics & 0.7 & 0.29 & 5.8 & 0.02 & 2 & $1.13-3.53$ \\
\hline Age, years & & & 138.1 & $<0.001$ & & \\
\hline$<44$ & & & & & 1 & \\
\hline $45-52$ & 0.53 & 0.28 & 3.63 & 0.06 & 1.7 & $0.99-2.93$ \\
\hline $53-65$ & 1.4 & 0.18 & 64.41 & $<0.001$ & 4.55 & $2.84-7.30$ \\
\hline $66+$ & 2.05 & 0.17 & 152.02 & $<0.001$ & 9.06 & $5.82-14.09$ \\
\hline
\end{tabular}

\begin{tabular}{|c|c|c|c|c|c|c|}
\hline Variable & Coefficient & s.e. & Wald & $P$ & RR & $95 \% \mathrm{Cl}$ \\
\hline Diagnosis of tardive dyskinesia & 0.11 & 0.134 & 0.657 & 0.418 & 1.12 & $0.86-1.45$ \\
\hline Use of conventional antipsychotics & 0.73 & 0.33 & 5 & 0.025 & 2.08 & $1.1-3.96$ \\
\hline Age, years & & & 131.58 & $<0.001$ & & \\
\hline$<44$ & & & & & 1 & \\
\hline $45-52$ & 0.54 & 0.28 & 3.75 & 0.053 & 1.71 & $1.00-2.95$ \\
\hline $53-65$ & 1.49 & 0.24 & 38.1 & $<0.001$ & 4.44 & $2.77-7.13$ \\
\hline $66+$ & 2.17 & 0.23 & 91.69 & $<0.001$ & 8.77 & $5.63-13.68$ \\
\hline
\end{tabular}


or from external causes. There were no significant differences in causes of death based on tardive dyskinesia status. Mortality rates per 100000 per year were calculated for each category of disease and are reported in Table 4.

\section{Discussion}

Despite the advent of atypical antipsychotics, the debate over the risk:benefit ratio of antipsychotics has become increasingly complex. ${ }^{13}$ This is due to the well-known neurological side-effects of conventional antipsychotics and the growing concerns over the metabolic side-effects of atypical antipsychotics, ${ }^{5,6}$ issues surrounding cost-effectiveness, ${ }^{14}$ and evidence indicating that atypical antipsychotics have little advantage over reasonably dosed conventional antipsychotics in drop-out rates as a result of adverse events, ${ }^{15}$ quality of life, ${ }^{14}$ efficacy, and rates of parkinsonism and tardive dyskinesia. ${ }^{14,15}$

The range of problems associated with both classes of drugs leaves even well-informed clinicians, patients and families still struggling with the risk:benefit ratio, and indeed facing an even greater degree of complexity than in the era preceding the widespread use of atypical antipsychotics. Adding to this debate has been the question of shortened survival time in those who develop tardive dyskinesia, but previous studies have been inconclusive. ${ }^{1}$ In this study, the initial increase in mortality found in those with tardive dyskinesia was overshadowed by the exposure to conventional antipsychotics only, which resulted in a significantly increased mortality rate compared with those taking only atypical antipsychotics. Not surprisingly, older age was a significant predictor of increased mortality, but there was no interaction between age and drug course.

Our data are supportive of a retrospective cohort study ${ }^{16}$ of first-time users of antipsychotics $(n=22890)$, aged 65 and older, in which those exposed to conventional antipsychotics had a $37 \%$ increase in mortality compared with those using atypical antipsychotics $(P=0.001)$. Perhaps most strikingly, the time to follow-up was 180 days or less, with the risk of death being significant at $<40$ days and at higher doses. Cancer, HIV infection and congestive heart failure were the only conditions conferring a greater adjusted risk than did conventional antipsychotics; however, the presence of antipsychotic-induced movement disorders was not described. The presence or absence of dementia was not associated with the increase in mortality.
Whether the use of conventional antipsychotics is a consistent predictor of shortened survival time is doubtful, since the use of either conventional antipsychotics or atypical antipsychotics in people with schizophrenia was associated with a significant, fourfold increase in mortality. ${ }^{17}$ In a cohort study of three US Medicaid programmes, ${ }^{18}$ the adjusted rate ratios for mortality, cardiac arrest and ventricular arrhythmias were higher for risperidone than haloperidol. However, the highest rate occurred in those receiving the lowest dose of risperidone, suggesting that this group may have been at higher risk and thus treated conservatively. Nevertheless, Saha et $a l^{19}$ in their systematic review of mortality in schizophrenia have emphasised the possibility that the increasing use of atypical antipsychotics and the development of the metabolic syndrome may be in part responsible for a striking increase in the median standardised mortality ratio (SMR) from 1.84 in the 1970s to 3.20 in the 1990s, despite the fact that in most countries age-standardised SMRs have fallen. Unfortunately, the authors found only three studies examining SMRs from lower income countries, but, given this cautionary note, they found no difference in SMRs in schizophrenia across sites.

\section{Complicating factors}

Since the population in this study is composed primarily of individuals with schizophrenia, we note that an excess mortality rate in this disorder was described as early as the 19th century, ${ }^{20}$ long before the introduction of biologically oriented therapies. This has continued, with the most recent analysis ${ }^{19}$ finding a median SMR of 2.58. Excessive deaths from both natural and unnatural causes have been widely recognised, with suicide being a prominent factor; ${ }^{19,21}$ however, others ${ }^{22}$ have found that death from unnatural causes was lower in schizophrenia compared with unipolar, bipolar and schizoaffective disorders but only in those aged 40-79 years. None the less, these authors found excessive mortality in all of the four diagnostic groups. In a meta-analytic study, ${ }^{20} 12 \%$ of all deaths and $28 \%$ of excess deaths were as a result of suicide, although deaths from unnatural causes were also prominent. Similarly, a study ${ }^{23}$ of first-admission patients admitted between 1970 and 1986 noted that of the 1100 people who died, half died by suicide.

These data are in marked contrast to our findings, wherein deaths by suicide and external causes were $6.8 \%$ and $4.9 \%$ respectively. However, the suicide rate in our study is 294.6 per 100000

\begin{tabular}{|c|c|c|c|c|c|c|c|c|}
\hline \multirow[b]{3}{*}{ Cause of death } & & & \multicolumn{6}{|c|}{ Antipsychotic } \\
\hline & \multicolumn{2}{|r|}{ Overall } & \multicolumn{2}{|r|}{ Atypical only } & \multicolumn{2}{|c|}{ Conventional only } & \multicolumn{2}{|c|}{ Atypical and conventional } \\
\hline & $n$ & $\begin{array}{c}\text { Rate per } 100000 \\
\text { per year }\end{array}$ & $n$ & $\begin{array}{c}\text { Rate per } 100000 \\
\text { per year }\end{array}$ & $n$ & $\begin{array}{l}\text { e per } 100000 \\
\text { per year }\end{array}$ & $n$ & $\begin{array}{c}\text { Rate per } 100000 \\
\text { per year }\end{array}$ \\
\hline Infection & 4 & 84.17 & 0 & & 3 & 80.32 & 1 & 158.37 \\
\hline Cancer & 42 & 883.84 & 0 & & 40 & 1070.96 & 2 & 316.73 \\
\hline Diabetes & 9 & 189.39 & 0 & & 9 & 240.97 & 0 & \\
\hline Heart disease/myocardial infarction & 49 & 1031.15 & 2 & 518.76 & 44 & 1178.06 & 3 & 475.11 \\
\hline Stroke/cerebrovascular disease & 17 & 357.74 & 0 & & 15 & 401.61 & 2 & 316.73 \\
\hline Influenza/pneumonia & 8 & 168.35 & 0 & & 8 & 214.19 & 0 & \\
\hline Chronic obstructive pulmonary disease & 12 & 252.53 & 0 & & 8 & 214.19 & 4 & 633.48 \\
\hline Liver disease & 2 & 42.09 & 1 & 259.38 & 1 & 26.77 & 0 & \\
\hline Accidents/external causes & 10 & 210.44 & 0 & & 9 & 240.97 & 1 & 158.37 \\
\hline Suicide & 14 & 294.61 & 0 & & 11 & 294.51 & 3 & 475.11 \\
\hline Other disease & 38 & 799.66 & 2 & 518.76 & 32 & 856.77 & 4 & 633.48 \\
\hline All causes & 205 & 4313.97 & 5 & 1296.91 & 180 & 4819.32 & 20 & 3167.4 \\
\hline
\end{tabular}


per year, similar to that described in other studies of schizophrenia. We also found substantial increases in mortality with age, in contrast to Brown, ${ }^{20}$ who noted a decline in mortality in schizophrenia with increasing age. Not surprisingly, others ${ }^{23,24}$ have found an increase, especially in deaths from cardiovascular disease.

The assessment of mortality in schizophrenia is also complicated by increased mortality rates in other psychiatric disorders (substance misuse, 'organic' mental disorders, eating disorders, major depression and others), ${ }^{22,25}$ many of which are comorbid with schizophrenia. We could not accurately assess these, since we did not use a structured diagnostic interview.

\section{Specific factors in antipsychotic-associated mortality}

In recent years there has been considerable interest in the effects of antipsychotics on cardiac status. One focus has been on rates of arrhythmia and arrest, ${ }^{18}$ with rate ratios of 1.3-3.2 in people with schizophrenia taking clozapine, haloperidol, risperidone or thioridazine. The rate ratios for death ranged from 2.6-5.8. However, the authors noted the difficulty in separating out treatment effects from the effects of schizophrenia. In another study, ${ }^{17}$ those using conventional antipsychotics had a five times greater risk of myocardial infarction than controls, but the risk was lower with greater intensity of antipsychotic use. Cardiomyopathy has been reported with fluphenazine, risperidone and lithium, and myocarditis with clozapine, lithium and chlorpromazine. ${ }^{26}$

Several other risk factors are worth noting. In a small study, ${ }^{27}$ antipsychotic polypharmacy was a risk factor for shortened survival time, as was being edentulous, an older male and not being treated with anticholinergics. A recent study ${ }^{28}$ found a $308 \%$ increase in the risk of colon cancer in people with schizophrenia taking antipsychotics, even when adjusted for smoking, obesity, comorbidity, concurrent medications and socio-economic status.

Finally, antipsychotics have been shown to induce cellular toxicity $^{29}$ via a number of mechanisms, including changes in proteins affecting cell survival, impairment of the mitochondrial respiratory chain, increases in DNA fragmentation and other changes in cell morphology. However, many studies have been short-term, and have often neglected important confounders such as polypharmacy and brain changes associated with comorbid disorders. It appears that we are badly in need of long-term studies of antipsychotic-induced neuronal changes and their influence on mortality. It does not appear, however, that the development of tardive dyskinesia carries an increased risk of mortality. Our study has several advantages over previous work, including a large population followed intensively over a decade by raters trained to reliability. This is the only study of mortality rates in tardive dyskinesia to utilise the National Death Index, which lent certainty to the fundamental question of whether a given individual had died during the observation period, in addition to giving us death certificate data on a large clinic population rated repeatedly for the presence of tardive dyskinesia. However, the demographics of the population may limit its applicability.

It appears that more attention needs to be given to discussions focusing on the risk:benefit ratio of antipsychotics. In years past, obtaining informed consent for the use of these agents has focused on the risks of incurring tardive dyskinesia and parkinsonism, but in recent years it has expanded to include the metabolic syndrome, with the associated possibility of a shortened lifespan. Is it now time to emphasise this possibility to an even greater degree, given the range of morbidity and mortality associated with these drugs?
Charles E. Dean, MD, Paul D. Thuras, PhD, Mental Health Service Line, Minneapolis Veterans Administration Hospital, Minneapolis, Minnesota, USA

Correspondence: Charles E. Dean, Mental Health Service Line, 116A One Veterans Drive, Minneapolis, MN 55417, USA. Email: charles.dean@med.va.gov

First received 17 Jan 2008, final revision 2 Sep 2008, accepted 14 Oct 2008

\section{Acknowledgements}

The authors would like to thank Ms Jean M. Russell for her work in data collection, rater training and in rating examinations performed in the Tardive Dyskinesia Assessment Clinic. We also thank Robert Bilgrad, of the Division of Vital Statistics, National Center for Health Statistics, for his help in completing the National Death Index application and making the data accessible and understandable.

\section{References}

1 Ballesteros J, Gonzalez-Pinto A, Bulbena A. Tardive dyskinesia associated with higher mortality in psychiatric patients: results of a meta-analysis of seven independent studies. J Clin Psychopharmacol 2000; 20: 188-94.

2 Janno S, Holi M, Tuisko K. Prevalence of neuroleptic-induced movement disorders in chronic schizophrenic patients. Am J Psychiatry 2004; 161: 160-3.

3 Owen RR, Feng W, Thrush CR, Hudson TJ, Austen MA. Variations in prescribing practices for novel antipsychotic agents among Veteran's Affairs Hospitals. Psychiatr Serv 2001; 52: 1523-5.

4 Allison DB, Mentore JL, Heo M, Chandler LP, Cappelleri JC, Infante MC, et al. Antipsychotic-induced weight gain: a comprehensive research synthesis. Am J Psychiatry 1999; 156: 1686-96.

5 McIntyre RS, McCann SM, Kennedy SH. Antipsychotic metabolic effects: weight gain, diabetes mellitus, and lipid abnormalities. Can J Psychiatry 2001; 46: 273-81.

6 Koro CE, Fedder DO, L'Italien GJ, Weiss S, Magder LS, Kreyenbuhl J, et al. An assessment of the independent effects of olanzapine and risperidone exposure on the risk of hyperlipidemia in schizophrenic patients. Arch Gen Psychiatry 2002; 59: 1021-6.

7 Sprague RL, Kalachnik JE, Breuning SE, Davis VJ, Ullmann RK, Cullari S, et al. The dyskinesia identification system-Coldwater (DIS-CO): a tardive dyskinesia rating system for the developmentally disabled. Psychopharmacol Bull 1984; 20: $328-38$.

8 Dean CE, Russell JR, Kuskowski MA, Caligiuri MP, Nugent SM. Clinical rating scales and instruments: how do they compare in assessing abnormal, involuntary movements? J Clin Psychopharmacol 2004; 24: 298-304.

9 Guy W. ECDEU Assessment Manual for Psychopharmacology: Publication $A D M$ 76-388. US Department of Health, Education, and Welfare, 1976.

10 Schooler NR, Kane JM. Research diagnoses for tardive dyskinesia (letter) Arch Gen Psychiatry 1995; 38: 336-9.

11 American Psychiatric Association. Diagnostic and Statistical Manual of Mental Disorders (4th edn) (DSM-IV). APA, 1994.

12 National Death Index. National Center for Health Statistics, 2002.

13 Abidi S, Bhaskara SM. From chlorpromazine to clozapine-antipsychotic adverse events and the clinician's dilemma. Can J Psychiatry 2003; 48: 749-55.

14 Rosenheck RA, Perlick D, Bingham S, Liu-Mares W, Collins J, Warren S, et al. Effectiveness and costs of olanzapine and haloperidol in the treatment of schizophrenia. JAMA 2003; 290: 2693-702.

15 Lieberman JA, Stroup TS, McEvoy JP. Effectiveness of antipsychotics drugs in patients with chronic schizophrenia. NEJM 2005; 353: 1209-23.

16 Wang PS, Schneeweiss S, Avorn J, Fischer MA, Mogun H, Solomon DH, et al. Risk of death in elderly uses of conventional antipsychotics vs atypical medications. N Engl J Med 2005; 353: 2335-41.

17 Enger C, Weatherby L, Reynolds RF, Glasser DB, Walker AM. Serious cardiovascular events and mortality among patients with schizophrenia. J Nerv Ment Dis 2004; 192: 19-27.

18 Hennessy S, Bilker WB, Knauss JS. Cardiac arrest and ventricular arrhythmia in patients taking antipsychotic drugs: cohort study using administrative data. BMJ 2002; 325: 1-5.

19 Saha S, Chant D, McGrath J. A systematic review of mortality in schizophrenia: is the mortality gap worsening over time? Arch Gen Psychiatry 2007; 64: 1123-31.

20 Brown S. Excess mortality in schizophrenia. A meta-analysis. Br J Psychiatry 1997; 171: 502-8. 
21 Allebeck $P$, Wisted B. Mortality in schizophrenia: a ten-year follow-up based on the Stockholm County Inpatient Register. Arch Gen Psychiatry 1986; 43 : 650-3.

22 Laursen TM, Munk-Olsen T, Nordentoft M, Mortensen PB. Increased mortality among patients admitted with major psychiatric disorders: a register-based study comparing mortality in unipolar depressive disorder, bipolar affective disorder, schizoaffective disorder, and schizophrenia. J Clin Psychiatry 2007 68: 899-907.

23 Mortensen $\mathrm{PB}$, Juel K. Mortality and causes of death in first admitted schizophrenic patients. Br J Psychiatry 1993; 163: 183-9.

24 Ösby $U$, Correia N, Ekbom A, Brandt L, Sparén P. Time trends in schizophrenia mortality in Stockholm County, Sweden: cohort study. BM. 2000; 321: 483-4

25 Harris EC, Barraclough B. Excess mortality of mental disorder. Br J Psychiatry 1998; 173: 11-53.
26 Coulter DM, Bate A, Meyboom RHB, Lindquist M, Edwards IR. Antipsychotic drugs in heart muscle disorder in international pharmacovigilance: data mining study. BMJ 2001; 322; 1207-9.

27 Waddington JL, Youseff HA, Kinsella A. Mortality in schizophrenia. Antipsychotic polypharmacy and absence of adjunctive anticholinergics over the course of a 10-year prospective study. Br J Psychiatry 1998; 173 $325-9$.

28 Hippisley-Cox J, Vinogradova Y, Coupland C, Parker, C. Risk of malignancy in patients with schizophrenia or bipolar disorder: nested case-control study. Arch Gen Psychiatry 2007; 64: 1368-76.

29 Dean CE. Antipsychotic-associated neuronal changes in the brain: toxic therapeutic, or irrelevant to the long-term outcome in schizophrenia? Prog Neuropsychopharmacol Biol Psychiatry 2006; 30: 174-89.

\section{Psychiatry in the movies}

\section{Watching zee detectives}

\section{Peter Byrne}

Psychiatrists ask a lot of questions. Had it not been already in existence (and practically sharing a birth date with cinema), filmmakers would have invented psychiatry to emote the 'Who?' 'What?' and 'How did you feel?' questions that must be asked to pause the action, explain and illuminate. It saves on intertitles or narration. If the film requires an authority figure, a psychiatrist will do nicely. There are many films which are not direct representations of the practice of psychiatry, but where an investigating psychiatrist carries the narrative - Blind Alley (1939) and Nightmare Alley (1947), to name only two. During the 1940s, therapists were featured in $4 \%$ of all US movies, but for film noir this figure rose to almost a quarter of all productions. Warner Baxter played Dr Robert Ordway (criminal turned psychiatrist) in as many as ten Crime Doctor films, beginning 1945. If for nothing else, we should celebrate the ordinariness of Ordway's name - think of Drs Max J. Englehoffer (Front Page, 1931), Otto von Strudel (Many Happy Returns, 1934), von Haller (Mr Deeds Goes to Town, 1936), Urganzieff (Bluebeard's Eighth Wife, 1938) and Kropotkin (A Fine Madness, 1966). The archetype was upgraded to Hitchcock's 'dream detectives' in Spellbound (1945), pictured. Though highly influential on movie psychiatry in general, and the representation of psychiatry as a bookish variant of detective work, Hitchcock described Spellbound as 'just another manhunt wrapped up in a lot of pseudo-psychoanalysis'.

Spellbound and the psychiatrist/detective had its immediate imitators - Dark Mirror (1946) and Dark Past (1948) - as well as influencing more modern films - They Might Be Giants (1971), Still of the Night (1982), Agnes of God (1985), Color of Night (1994) and Copycat (1995) - but it is difficult to cite more recent examples (one of the few would be Borderline, 2002). Admittedly, 12 Monkeys (1995) though paying homage to Vertigo (1957), owes far more to the detective storyline of Hitchcock's 1945 classic. However, despite a strong cast and talented director, 12 Monkeys failed to achieve commercial or critical success. Cinema may have played out the old-fashioned idea of a psychiatrist/detective.

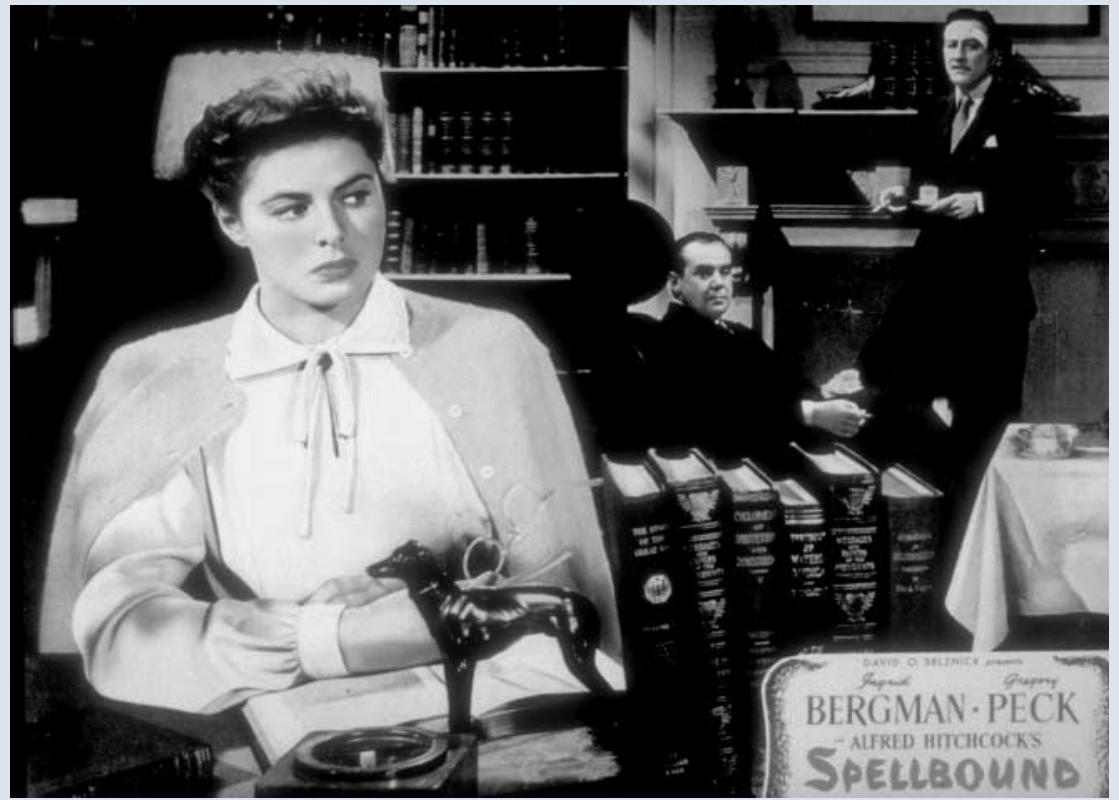

Still from Spellbound; image courtesy London Features International. 